\title{
Time-Optimal Control for Semilinear Stochastic Functional Differential Equations with Delays
}

\author{
Yong Han Kang ${ }^{1}$ and Jin-Mun Jeong ${ }^{2, *(D)}$ \\ 1 Institute of Liberal Education, Catholic University of Daegu, Gyeongsan 38430, Korea; yonghann@cu.ac.kr \\ 2 Department of Applied Mathematics, Pukyong National University, Busan 48513, Korea \\ * Correspondence: jmjeong@pknu.ac.kr
}

Citation: Kang, Y.H.; Jeong, J.-M. Time Optimal Control for Semilinear Stochastic Functional Differential Equations with Delays. Mathematics 2021, 9, 1956. https://doi.org/ $10.3390 /$ math 9161956

Academic Editor: Eva H. Dulf

Received: 11 May 2021

Accepted: 9 August 2021

Published: 16 August 2021

Publisher's Note: MDPI stays neutral with regard to jurisdictional claims in published maps and institutional affiliations.

Copyright: (c) 2021 by the authors. Licensee MDPI, Basel, Switzerland. This article is an open access article distributed under the terms and conditions of the Creative Commons Attribution (CC BY) license (https:// creativecommons.org/licenses/by/ $4.0 /)$.

\begin{abstract}
The purpose of this paper is to find the time-optimal control to a target set for semilinear stochastic functional differential equations involving time delays or memories under general conditions on a target set and nonlinear terms even though the equations contain unbounded principal operators. Our research approach is to construct a fundamental solution for corresponding linear systems and establish variations of a constant formula of solutions for given stochastic equations. The existence result of time-optimal controls for one point target set governed by the given semilinear stochastic equation is also established.
\end{abstract}

Keywords: stochastic differential equation; retarded control system; time-optimal control; admissible set; analytic semigroup.

\section{Introduction}

This paper deals with the existence of optimal control to reach the target set governed by semilinear stochastic differential equations:

$$
\left\{\begin{array}{l}
x^{\prime}(t)=A x(t)+\int_{-h}^{0} a_{1}(s) A_{1} x(t+s) d s+f\left(t, x_{t}\right) d \omega+B u(t), \quad t>0, \\
x(0)=\phi^{0} \in L^{2}(\Omega, H), \quad x(s)=\phi^{1}(s), \quad s \in[-h, 0],
\end{array}\right.
$$

where $A$ is an elliptic differential operator of the second order induced by the sesquilinear form, $A_{1}$ is a closed linear operator with domain $D\left(A_{1}\right)$ containing the $D(A), h>0$, and the function $a(\cdot)$ is real-valued and Hölder-continuous. Moreover, $\omega(t)$ represents a $\mathrm{K}$-valued Brownian motion or Wiener process with a finite trace nuclear covariance operator $Q$, and $f$ is a nonlinear mapping satisfying some assumptions. Let $U$ be a Banach space as a control space, and the controller $B$ be linear-bounded from $U$ to $H$.

The purpose of this paper is to find the time-optimal control governed by (1) to a bounded target set in the shortest time. This kind of stochastic differential equation arises in many practical mathematical models, such as option pricing, population dynamics, physical, engineering, and biological problems, and so forth (see [1-4]). Many studies in the literature have been studied for the theory of stochastic differential equations in a variety of ways in [5,6] and the reference therein. The approximate controllability of stochastic equations have been studied by the authors [7-10]. Similar considerations of semilinear stochastic systems have been dealt with in many references [11,12].

Some standard optimal control results for general linear systems with delays in reflexive Banach spaces were studied in [13]. Recently, a survey of results on optimal control problems governed by delay differential inclusions was referred to by Mordukhovich et al. [14,15], and [16,17]. Micu et al. [18], who discussed the time-optimal boundary controls for the heat equation, and the parabolic equations with the Neumann condition was considered by Krakowiak [19]. The optimal control problems of retarded linear systems with bounded operators have been extensively studied by $[13,20]$. If the principal operator is unbounded, 
Jeong and Son [21] investigated the time-optimal control results to a target set for semilinear control equations involving time delay. However, little study has been done of the existence of time-optimal control for control to a target set for nonlinear stochastic functional differential equations with unbounded operators.

In case (1), since the mild solution has discontinuities at delay times, we have difficulty inducing the basic properties of solutions of semilinear stochastic control systems. However, by interpreting the fundamental solutions for linear functional equations with time delay as seen in [22], we can overcome the difficulty and obtain some essential results of solutions of stochastic differential equations with delays in Hilbert spaces. Moreover, motivated by the above-mentioned works $[13,21]$, we deal with the time-optimal control problem to a bounded target set for semilinear stochastic control equations involving time delays or memories even though the equations contain unbounded principal operators and nonlinear terms by using an easy consequence of real interpolation spaces.

We enumerate the contents of this paper. Section 2 introduces some basic results on the general retarded linear equations by constructing the fundamental solution, and deal with a variation of a constant formula of $L^{2}$-primitive processes and properties of the strict solutions of (1). In Section 3, we deal with the existence of optimal control to reach the target set governed by semilinear stochastic differential equations. As the time-optimal control theory for the standard results, we refer to the linear case as in [13] (or semilinear equation [21]) and extend the results in our semilinear stochastic functional differential equations with delays. Finally, the existence of the optimal control to a singleton target is also derived from the convergence of optimal controls to decreasing target sets containing the singleton.

\section{Preliminaries and Lemmas}

\subsection{Retarded Linear Equations}

Let $H$ be a Hilbert space that is densely embedded, and $V$ be continuously embedded in $H$. The norms of $V, H$, and the dual space $V^{*}$ with $V$ are denoted by $\|\cdot\|,|\cdot|$, and $\|\cdot\|_{*}$, respectively. It can generally be considered that

$$
\|u\|_{*} \leq|u| \leq\|u\|, \quad u \in V .
$$

If $b(\cdot, \cdot)$ is a continuous sesquilinear form in $V \times V$ satisfying Gårding's inequality:

$$
\operatorname{Re} b(u, u) \geq c_{0}\|u\|^{2}-c_{1}|u|^{2}, \quad c_{0}>0, \quad c_{1} \geq 0 .
$$

We define $A$ as follows:

$$
<\left(c_{1}-A\right) u, v>=-b(u, v), \quad u, v \in V,
$$

where $<\cdot, \cdot>$ also denotes the duality pairing between $V$ and $V^{*}$. By (2), we have

$$
\operatorname{Re}\left\langle A u, u>\geq c_{0}\|u\|^{2}, \quad u \in V .\right.
$$

According to the Lax-Milgram theorem, we know that $A$ is a bounded linear operator from $V$ to $V^{*}$. Moreover, as seen in Theorem 3.6.1 of [20], $A$ generates an analytic semigroup $S(t)=e^{t A}$ in both $H$ and $V^{*}$. The restriction of $A$ to domain

$$
D(A)=\{u \in V ; A u \in H\}
$$

with the graph norm is also denoted by $A$. By identifying the dual of $H$ with $H$, we may consider the following relation

$$
D(A) \subset V \subset H \subset V^{*} \subset D(A)^{*} .
$$


Let $(D(A), H)_{1 / 2,2}$ be the real intermediate space between $D(A)$ and $H$ in the sense of Section 1.3.3 of [23]. In relation to (3), it is well-known that

$$
\left(V, V^{*}\right)_{1 / 2,2}=H, \quad(D(A), H)_{1 / 2,2}=V .
$$

If $X$ and $Y$ are two Banach spaces, $\mathcal{L}(X, Y)$ is the collection of all bounded linear operators from $X$ into $Y$, and $\mathcal{L}(X, X)$ is simply written as $\mathcal{L}(X)$. For simplicity, we assume that $S(t)$ is uniformly bounded, that is, there exists a constant $C_{0}>0$ such that

$$
\|S(t)\|_{\mathcal{L}(H)} \leq C_{0} .
$$

Lemma 1. Let $S(t)$ be an analytic semigroup generated by $A$. Then we have

$$
\|S(t)\|_{\mathcal{L}(H)} \leq C_{0}, \quad\|A S(t)\|_{\mathcal{L}(H)} \leq \frac{C_{0}}{t}, \quad \text { and }\left\|A^{2} S(t)\right\|_{\mathcal{L}(H)} \leq \frac{C_{0}}{t^{2}}
$$

for each $t \geq 0$ (e.g., [20]), and for $0<s<t$ and $0<\alpha<1$

$$
\|S(t)-S(s)\| \leq \frac{C_{0}}{\alpha}(t-s)^{\alpha} s^{-\alpha} .
$$

Proof. For $0<s<t$

$$
\|S(t)-S(s)\|=\int_{0}^{t} A S(\tau) d \tau \leq C_{0} \log \left(\frac{t}{S}\right),
$$

noting that for $0<\alpha<1$

$$
\log \left(\frac{t}{s}\right)=\int_{1}^{1+\frac{t-s}{s}} \frac{1}{y} d y \leq \int_{1}^{1+\frac{t-s}{s}} \frac{1}{y^{1-\alpha}} d y \leq \frac{1}{\alpha}\left(\frac{t-s}{s}\right)^{\alpha},
$$

combining this and (5), we get (4) for $0<s<t$ and $0<\alpha<1$.

Now, consider the following retarded linear functional differential equation:

$$
\left\{\begin{array}{l}
x^{\prime}(t)=A x(t)+\int_{-h}^{0} a(s) A_{1} x(t+s) d s+k(t), \quad t>0, \\
x(0)=\phi^{0}, \quad x(s)=\phi^{1}(s) \quad-h \leq s \leq 0,
\end{array}\right.
$$

where $A_{1}$ is a closed linear unbounded operator with $D(A) \subset D\left(A_{1}\right)$, for instance, $A_{1}$ is an elliptic differential operator of second order induced by sesquilinear form. As seen in Harakiri [24], we introduce the fundamental solution $W(\cdot)$ of the retarded linear Equation (6) defined by

$$
\left\{\begin{array}{l}
W(t)=S(t)+\int_{0}^{t} S(t-s)\left\{\int_{-h}^{0} a(\tau) A_{1} W(s+\tau) d \tau\right\} d s, \quad t>0 . \\
W(0)=I, \quad W(s)=0, \quad-h \leq s<0
\end{array}\right.
$$

where $a(\cdot)$ is a real-valued, Hölder-continuous function:

$$
|a(s)| \leq H_{0}, \quad|a(s)-a(\tau)| \leq H_{0}(s-\tau)^{\rho}, \quad-h \leq \tau, s \leq 0
$$

for a constant $H_{0}$.

By the property of $S(\cdot)$ as in Lemma $1, W(t)$ is strongly continuous in both $H$ and $V^{*}$. For each $t>0$, if we define the operator $U_{t}(\cdot)$ by

$$
U_{t}(s)=\int_{-h}^{s} W(t-s+\sigma) a(\sigma) A_{1} d \sigma: V \rightarrow V, \quad s \in[-h, 0] .
$$


Then, (4) is represented by

$$
x(t)=W(t) \phi^{0}+\int_{-h}^{0} U_{t}(s) \phi^{1}(s) d s+\int_{0}^{t} W(t-s) k(s) d s .
$$

By Lemma 1, we obtain basic properties of $W(t)$ (see Proposition 4.1 of [25] or Theorem 1 of [22]).

Lemma 2. The fundamental solution $W(t)$ exists uniquely, and there exists a constant $C_{0}>0$ such that

$$
\begin{aligned}
& \|W(t)\|_{\mathcal{L}(H)}(t \geq 0) \leq C_{0},\|W(t)\|_{\mathcal{L}\left(V^{*}\right)}(t \geq 0) \leq C_{0}, \\
& \left\|W\left(t^{\prime}\right)-W(t)\right\|_{\mathcal{L}(H)} \leq \frac{C_{0}}{\alpha}\left(t^{\prime}-t\right)^{\alpha} t^{-\alpha}, \quad t>0, \\
& \left\|W\left(t^{\prime}\right)-W(t)\right\|_{\mathcal{L}\left(V^{*}\right)} \leq C_{0}\left(t^{\prime}-t\right), \\
& \left\|W\left(t^{\prime}\right)-W(t)\right\|_{\mathcal{L}\left(V^{*}, V\right)} \leq C_{0}\left(t^{\prime}-t\right)^{\kappa}(t-h)^{-\kappa}
\end{aligned}
$$

for $h<t<t^{\prime}$, and $\kappa<\rho$, where $\rho$ is the Hölder constant in (7).

\subsection{Semilinear Stochastic Differential Equations}

Let $(H,|\cdot|)$ and $\left(K,|\cdot|_{K}\right)$ be real separable Hilbert spaces. Consider the following retarded semilinear stochastic control system in Hilbert space $H$ :

$$
\left\{\begin{array}{l}
x^{\prime}(t)=A x(t)+\int_{-h}^{0} a_{1}(s) A_{1} x(t+s) d s+f\left(t, x_{t}\right) d \omega+B u(t), \quad t>0, \\
x(0)=\phi^{0} \in L^{2}(\Omega, H), \quad x(s)=\phi^{1}(s), \quad s \in[-h, 0] .
\end{array}\right.
$$

Let $(\Omega, \mathcal{F}, P)$ be a complete probability space with a complete family of right-continuous increasing sub $\sigma$-algebras $\left\{\mathcal{F}_{t}, t \in I\right\}$ satisfying $\mathcal{F}_{t} \subset \mathcal{F}$.

The collection of random variables $\mathcal{S}=\{\mathcal{F}$-measurablefunction $x(t, w): \Omega \rightarrow H:$ $t \in[0, T], w \in \Omega\}$ is a stochastic process. Generally, we just write $x(t)$ instead of $x(t, w)$ and $x(t):[0, T] \rightarrow H$ in the space of $\mathcal{S}$.

Let $\left\{e_{n}\right\}_{n=1}^{\infty}$ be a complete orthonormal basis of $K$, and let $Q \in \mathcal{L}(K)$ be an operator defined by $Q e_{n}=\lambda_{n} e_{n}$ with finite $\operatorname{Tr}(Q)=\sum_{n=1}^{\infty} \sqrt{\lambda_{n}}=\lambda<\infty$ (Tr denotes the trace of the operator), where $\lambda_{n} \geq 0(n=1,2, \cdots)$. Here, $\mathcal{L}(K, H)$ denotes the space of all bounded linear operators from $K$ into $H$, and we denote simply $\mathcal{L}(K)$ if $H=K$.

Let $\{\omega(t): t \geq 0\}$ be a cylindrical $K$-valued Wiener process with a finite trace nuclear covariance operator $Q$ over $(\Omega, \mathcal{F}, P)$, which satisfies that

$$
\omega(t)=\sum_{n=1}^{\infty} \sqrt{\lambda_{n}} w_{i}(t) e_{n}, \quad t \geq 0,
$$

where $\left\{w_{i}(t)\right\}_{i=1}^{\infty}$ is a mutually independent, one-dimensional standard Wiener process over $(\Omega, \mathcal{F}, P)$. Then, the above $K$-valued stochastic process $\omega(t)$ is called a $Q$-Wiener process.

We assume that $\mathcal{F}_{t}=\sigma\{\omega(s): 0 \leq s \leq t\}$ is the $\sigma$-algebra generated by $w$ and $\mathcal{F}_{T}=\mathcal{F}$. Let $\psi \in \mathcal{L}(K, H)$, and define

$$
|\psi|_{Q}^{2}=\operatorname{Tr}\left(\psi Q \psi^{*}\right)=\sum_{n=1}^{\infty}\left|\sqrt{\lambda_{n}} \psi e_{n}\right|^{2} .
$$

If $|\psi|_{Q}^{2}<\infty$, then $\psi$ is called a $Q$-Hilbert-Schmidt operator. $\mathcal{L}_{Q}(K, H)$ stands for the space of all $Q$-Hilbert-Schmidt operators. The completion $\mathcal{L}_{Q}(K, H)$ of $\mathcal{L}(K, H)$ with respect to the topology induced by the norm $|\psi|_{Q}$, where $|\psi|_{Q}^{2}=(\psi, \psi)$ is a Hilbert space with the above norm topology. 
Assume that $V$ is s dense space of $H$, as seen in Section 2.1. For $T>0$, we define

$$
M^{2}(-h, T ; V)=\left\{x:[-h, T] \rightarrow V: E\left(\int_{-h}^{T}\|x(s)\|^{2} d s\right)<\infty\right\} .
$$

The spaces $M^{2}(-h, 0 ; V), M^{2}(0, T ; V)$, and $M^{2}\left(0, T ; V^{*}\right)$ are also defined as the same way, and the basic theory of $M_{2}$-spaces can be found in [2].

For $h>0$, we assume that $\phi^{1}:[-h, 0) \rightarrow V$ is a given initial value satisfying

$$
E\left(\int_{-h}^{0}\left\|\phi^{1}(s)\right\|^{2} d s\right)<\infty
$$

that is, $\phi^{1} \in M^{2}(-h, 0 ; V)$. On this note, a random variable $x(t): \Omega \rightarrow H$ will be called an $L^{2}$-primitive process if $x \in M^{2}(-h, T ; V)$.

For every $s \in[0, T]$, we define $x_{s}:[-h, 0] \rightarrow H$ as

$$
x_{s}(r)=x(s+r), \quad-h \leq r \leq 0 .
$$

For brevity, we will set

$$
\Pi=M^{2}(-h, 0 ; V)
$$

Definition 1. A stochastic process $x:[-h, T] \times \Omega \rightarrow H$ is called a solution of (9) if

(i) $\quad x(t)$ is measurable and $\mathcal{F}_{t}$-adapted for each $t \geq 0$.

(ii) $\quad x(t) \in H$ has cádlág paths on $t \in(0, T)$ such that

$$
x(t)=W(t) \phi^{0}+\int_{-h}^{0} U_{t}(s) \phi^{1}(s) d s+\int_{0}^{t} W(t-s)\left\{f\left(s, x_{s}\right) d \omega+B u(s)\right\} d s,
$$

where

$$
U_{t}(s)=\int_{-h}^{s} W(t-s+\sigma) a(\sigma) A_{1} d \sigma .
$$

(iii) $x \in M^{2}(0, T ; V)$, that is, $E\left(\int_{0}^{T}\|x(s)\|^{2} d s\right)<\infty$ and $x \in C([0, T] ; H)$.

To get our results, we need the following assumptions on (9).

Assumption 1. Let $f: \mathbb{R} \times \Pi \rightarrow \mathcal{L}(K, H)$ be a nonlinear mapping satisfying the following:

(i) For each $x \in \Pi$, the mapping $f(\cdot, x)$ is strongly measurable.

(ii) There is a function $L_{f}: \mathbb{R}_{+} \rightarrow \mathbb{R}$ such that

$$
\begin{aligned}
& \qquad \begin{array}{l}
E f(t, x)-\left.f(t, y)\right|^{2} \leq L_{f}(r)\|x-y\|_{\Pi}^{2}, \quad t \in[0, T] \\
E|f(t, x)|^{2} \leq L_{f}(r)\left(\|x\|_{\Pi}+1\right)^{2}
\end{array} \\
& \text { for }\|x\|_{\Pi} \leq \text { rand }\|y\|_{\Pi} \leq r .
\end{aligned}
$$

Let the solution spaces $\mathcal{Z}(T)$ and $\mathcal{Z}_{0}(T)$ be defined by

$$
\begin{aligned}
& \mathcal{Z}(T):=M^{2}(0, T ; V) \cap C([0, T] ; H), \\
& \mathcal{Z}_{0}(T):=M^{2}(0, T ; D(A)) \cap C([0, T] ; V) .
\end{aligned}
$$

We can briefly summarize about the solvability of the system (9) from [26].

Proposition 1. 1) Let Assumption (F) be satisfied. Suppose that $\left(\phi^{0}, \phi^{1}\right) \in L^{2}(\Omega, H) \times \Pi$ and $k \in M^{2}\left(0, T ; V^{*}\right)$ for $T>0$. Then, there is a solution $x$ of the system (9) such that

$$
x \in \mathcal{Z}(T) .
$$


Moreover, there is a positive constant $C_{1}$ independent of the initial data $\left(\phi^{0}, \phi^{1}\right)$ and the forcing term $k$ such that

$$
\|x\|_{\mathcal{Z}(T)} \leq C_{1}\left(1+E\left(\left|\phi^{0}\right|^{2}\right)+\left\|\phi^{1}\right\|_{\Pi}+\|k\|_{M^{2}\left(0, T ; V^{*}\right)}\right) .
$$

2) Let Assumption (F) be satisfied. Suppose that the initial data $\left(\phi^{0}, \phi^{1}\right) \in L^{2}(\Omega, H) \times \Pi$ and the forcing term $k \in M^{2}\left(0, T ; V^{*}\right)$. Then the solution $x$ of the Equation (9) belongs to $x \in M^{2}(0, T ; V)$ and the mapping

$$
L^{2}(\Omega, H) \times \Pi \times M^{2}\left(0, T ; V^{*}\right) \ni\left(\phi^{0}, \phi^{1}, k\right) \mapsto x \in M^{2}(0, T ; V)
$$

be continuous.

\section{Time-Optimal Control Problems}

Let $U$ be a Banach space as a control space and the controller $B \in \mathcal{L}(U, H)$. The solution $x(t)=x(t ; \phi, F, u)$ of the system (9) is the following form:

$$
\left\{\begin{array}{l}
x(t ; \phi, f, u)=W(t) \phi^{0}+\int_{-h}^{0} U_{t}(s) \phi^{1}(s) d s+\int_{0}^{t} W(t-s)\left\{f\left(s, x_{s}\right) d \omega+B u(s) d s\right\} \\
u \in U_{a d}
\end{array}\right.
$$

where

$$
U_{t}(s)=\int_{-h}^{s} W(t-s+\sigma) a(\sigma) A_{1} d \sigma,
$$

$A_{1}$ is the operator mentioned in Section 2, and $U_{a d}$ is a class of admissible controls. For $x_{t} \in \Pi$ and $(t, s) \in[0, \infty) \times[-h, 0]$, we set

$$
f\left(t, x_{t}\right)=\int_{-h}^{0} g\left(t, s, x_{t}(s)\right) d \mu
$$

We need the following assumptions on the nonlinear operator $f$ :

Let $\mathcal{A}$ and $\mathcal{B}$ be the Lebesgue $\sigma$-field on $[0, \infty[$ and the Borel $\sigma$-field on $[-h, 0]$, respectively. Let $\mu$ be a Borel measure on $[-h, 0]$.

Assumption 2. Let $g$ be a nonlinear mapping from $[0, \infty[\times[-h, 0] \times V$ to $\mathcal{L}(K, H)$ satisfying the following:

(i) For any $x \in V$, the mapping $g(\cdot, \cdot, x)$ is strongly $\mathcal{A} \times \mathcal{B}$-measurable, and $x \mapsto g(\cdot, \cdot, x)$ is compact;

(ii) $g(t, s, x)$ is Lipschitz-continuous in $x$, uniformly in $t$ and $s$, that is, there exist a positive constant $L_{1}$ such that

$$
\begin{aligned}
& E|g(t, s, x)|^{2} \leq L_{1}(1+|| x||)^{2}, \\
& E|g(t, s, x)-g(t, s, \hat{x})|^{2} \leq L_{1} E|| x-\hat{x} \|^{2},
\end{aligned}
$$

for all $(t, s) \in[0, \infty[\times[-h, 0]$ and $x, \hat{x} \in V$.

Remark 1. The nonlinear operator $g$ mentioned above is related with the semilinear case of the nonlinear part of quasilinear equations introduced by Yong and Pan [27].

Now, we will establish the time-optimal control problem governed by (10) in $H$. Throughout this section, let the admissible set $U_{a d}$ be a weakly compact subset in $L^{2}(0, T ; U)$. We sometimes represent the solution $x(t)$ in (10) by $x_{u}(t)$ to express the dependence on $u \in U_{a d}$. The function $x_{u}$ is called the trajectory corresponding to a control $u$. Let the target set $W$ be bounded in $H$. Define

$$
U_{0}=\left\{u \in U_{a d}: x_{u}(t) \in W \text { for some } t \in[0, T]\right\}
$$


and assume that $U_{0} \neq \varnothing$. Then, a control $u \in U_{0}$ is equivalent that there is a $u \in U_{a d}$ such that for some $w \in W$

$$
E\left|w-x_{u}(t)\right|=0 .
$$

The optimal time is defined by a low limit $t_{0}$ of

$$
\left\{t: x_{u}(t) \in W \text { for some } u \in U_{0}\right\} .
$$

For every $u \in U_{0}$, we are able to define the shortest time $\tilde{t}(u)$ such that $x_{u}(\tilde{t}) \in W$. Our purpose is to seek a $\bar{u} \in U_{0}$ satisfying

$$
\tilde{t}(\bar{u}) \leq \tilde{t}(u) \quad \text { for all } u \in U_{0}
$$

constrained to the system (10). Since $x_{u} \in C([0, T] ; H)$, the transition time $\tilde{t}(u)$ is welldefined for each $u \in U_{a d}$. Finally, the existence of the optimal control to a singleton target is also derived from the convergence of optimal controls to decreasing target sets containing the singleton.

We define the linear operators $K_{1}$ from $M^{2}(0, T ; V)$ to $H$ and $K_{2}$ from $\mathcal{L}(K, H)$ to $H$ by

$$
\begin{array}{ll}
K_{1}(t) p=\int_{0}^{t} W(t-s) p(s) d s, & t \leq T, \\
K_{2}(t) q=\int_{0}^{t} W(t-s) q(s) d \omega, & t \leq T,
\end{array}
$$

respectively.

Lemma 3. Let $x \in M^{2}(0, T ; V)$. Then, for $0 \leq t \leq T, x_{t} \in \Pi$ and $f\left(\cdot, x_{t}\right) \in M^{2}(0, T ; H)$ and

$$
\|\left. f(\cdot, x .)\right|_{M^{2}(0, T ; H)} \leq \mu([-h, 0])\left\{L_{0} \sqrt{T}+L_{1}\|x\|_{M^{2}(0, T ; V)}+L_{1}\|x\|_{\Pi}\right\} .
$$

Moreover, if $x^{1}, x^{2} \in M^{2}(0, T ; V)$, then

$$
\left\|f\left(\cdot, x^{1}\right)-f\left(\cdot, x^{2}\right)\right\|_{M^{2}(0, T ; H)} \leq \mu([-h, 0]) L_{1}\left\{\left\|x_{1}-x_{2}\right\|_{M^{2}(0, T ; H)}+\left\|x_{1}-x_{2}\right\|_{M^{2}(-h, 0 ; H)}\right\} .
$$

Proof. Noting that

$$
\left\|x_{t}\right\|_{\Pi}^{2}=E\left(\int_{-h}^{0}\|x(t+\tau)\|^{2} d \tau\right) \leq E\left[\int_{-h}^{t}\|x(\tau)\|^{2} d \tau\right] \leq\|x\|_{M^{2}(-h, t ; V)}^{2}, t>0,
$$

from (ii) of Assumption (G), we have

$$
\begin{aligned}
\left\|f\left(\cdot, x_{.}^{1}\right)-f\left(\cdot, x_{.}^{2}\right)\right\|_{M^{2}(0, T ; H)} & \leq \mu([-h, 0]) L_{1}\left\|x_{t}^{1}-x_{t}^{2}\right\|_{\Pi} \\
& \leq \mu([-h, 0]) L_{1}\left\|x^{1}-x^{2}\right\|_{M^{2}(-h, T ; V)} .
\end{aligned}
$$

The first paragraph is similar.

By virtue of Lemma 3, from Proposition 1, it follows that a solution $x$ of (10) exists in $\mathcal{Z}(T)$. Now, we find a time-optimal control which transfers from the initial data to the target set to the trajectory of the constraint system (10) in the first time as follows.

Theorem 1. Let Assumption $(G)$ be satisfied and $U_{0} \neq \varnothing$. Then there exists a time-optimal control.

Proof. Let $t_{n} \downarrow t_{0}$ as $n \rightarrow \infty, u_{n} \in U_{a d}$, and suppose that $x_{n} \in W$. Since $U_{a d}$ is weakly compact, there is an $u_{0} \in U_{a d}, \hat{x} \in W$ and a subsequence, which is denoted again by $\left\{u_{n}\right\}$ and $\left\{x_{n}\left(t_{n}\right)\right\}$ such that $u_{n} \rightarrow u_{0}$ weakly in $L^{2}(0, T ; Y)$, that is, $w-\lim _{n \rightarrow \infty} u_{n}=u_{0}$ and $x_{n}\left(t_{n}\right) \rightarrow \hat{x}$ weakly in $W$. 
Let $\mathcal{F}$ and $\mathcal{B}$ be the Nijinsky operators corresponding to the maps $f$ and $\mathcal{B}$, which are defined by

$$
(\mathcal{F} u)(\cdot)=f\left(\cdot, x_{u}\right), \quad \text { and } \quad \mathcal{B} u(\cdot)=B u(\cdot),
$$

respectively. Then

$$
x_{n}\left(t_{n}\right)=x\left(t_{n} ; \phi, 0\right)+K_{2}\left(t_{n}\right) \mathcal{F} u_{n}+K_{1}\left(t_{n}\right) \mathcal{B} u_{n}
$$

where

$$
x\left(t_{n} ; \phi, 0\right)=W\left(t_{n}\right) \phi^{0}+\int_{-h}^{0} U_{t_{n}}(s) \phi^{1}(s) d s,
$$

and $K_{1}$ and $K_{2}$ are the operators defined by (11) and (12), respectively. From the strong continuity of $W(t)$, it follows that

$$
x\left(t_{n} ; \phi, 0\right) \rightarrow x\left(t_{0} ; \phi, 0\right) \text { strongly in } H .
$$

By the property of $W(t)$, see $[24,25,28]$, we have

$$
W\left(t_{n}-s\right)=S\left(t_{n}-t_{0}\right) W\left(t_{0}-s\right)+\int_{t_{0}-s}^{t_{n}-s} S\left(t_{n}-s-\sigma\right) \int_{-h}^{0} a(\tau) A_{2} W(s+\tau) d \tau d \sigma .
$$

Hence,

$$
\begin{aligned}
E\left|K_{2}\left(t_{n}\right) \mathcal{F} u_{n}-K_{2}\left(t_{0}\right) \mathcal{F} u_{0}\right| \leq & E\left|\int_{t_{0}}^{t_{n}} W\left(t_{n}-s\right)\left(\mathcal{F} u_{n}\right)(s) d \omega\right| \\
& +E\left|\int_{0}^{t_{0}}\left(W\left(t_{n}-s\right)-W\left(t_{0}-s\right)\right)\left(\mathcal{F} u_{n}\right)(s) d \omega\right| \\
& +E\left|\int_{0}^{t_{0}} W\left(t_{0}-s\right)\left(\left(\mathcal{F} u_{n}\right)(s)-\left(\mathcal{F} u_{0}\right)(s)\right) d \omega\right| \\
= & I+I I+I I I .
\end{aligned}
$$

By Assumption (G), we get

$$
I \leq \sqrt{t_{n}-t_{0}} C_{0} L_{f}(r) \operatorname{Tr}(Q)\left(\left\|x_{u}\right\|_{\Pi}+1\right),
$$

and by (8),

$$
I I \leq C_{0} \alpha^{-1}(1-2 \alpha)^{-1 / 2}\left(t_{n}-t_{0}\right)^{\alpha} t^{(1-2 \alpha) / 2} \operatorname{Tr}(Q) \mu([-h, 0])\left(L_{0} \sqrt{t}+L_{1}\left\|x_{u}\right\|_{M^{2}(-h, t ; V)}\right) .
$$

Hence, $I, I I \rightarrow 0$ as $n \rightarrow \infty$ if $\alpha<1 / 2$. Since $\mathcal{F}$ is compact, we have that $\mathcal{F} u_{n} \rightarrow \mathcal{F} u_{0}$ tends to zero as $t_{n} \downarrow t_{0}$, so are III and (13) by (14)-(17).

Now we will show that $K_{1}$ is a completely continuous mapping. If so, then from the formula

$$
\begin{aligned}
E\left|K_{1}\left(t_{n}\right) \mathcal{B} u_{n}-K_{1}\left(t_{0}\right) \mathcal{B} u_{0}\right|= & E\left|\int_{t_{0}}^{t_{n}} W\left(t_{n}-s\right)\left(\mathcal{B} u_{n}\right)(s) d s\right| \\
& +E\left|\int_{0}^{t_{0}}\left(W\left(t_{n}-s\right)-W\left(t_{0}-s\right)\right)\left(\mathcal{B} u_{n}\right)(s) d s\right| \\
& +E\left|\int_{0}^{t_{0}} W\left(t_{0}-s\right)\left(\left(\mathcal{B} u_{n}\right)(s)-\left(\mathcal{B} u_{0}\right)(s)\right) d s\right|
\end{aligned}
$$

it holds that

$$
E\left|K_{1}\left(t_{n}\right) \mathcal{B} u_{n}-K_{1}\left(t_{0}\right) \mathcal{B} u_{0}\right|=0
$$

as $t_{n} \downarrow t_{0}$ in a similar way to (15). In order to proof of compactness of $K_{1}$, we will show that

$$
\lim _{n \rightarrow \infty}\left|K_{1} \mathcal{B} u_{n}-K_{1} \mathcal{B} u_{0}\right|=0,
$$


which means that the completely continuity of $K_{1}$, since $H$ is reflexive. Set

$$
(h u)(s)=W\left(t_{0}-s\right)(\mathcal{B} u)(s) .
$$

Then by (6), we have

$$
|h(s)| \leq C_{0}|| B\left\|_{(Y, H)}|| u_{n}(s)-u_{0}\right\|_{Y} \leq \infty
$$

For a fixed $s \in\left[0, t_{0}\right]$, let $x_{s}^{*}(u)=(h u)(s)$ for every $u \in L^{2}\left(0, T_{1} ; Y\right)$. Then $x_{s}^{*} \in L^{2}\left(0, t_{0} ; Y^{*}\right)$, and we have $\lim _{n \rightarrow \infty} x_{s}^{*}\left(u_{n}\right)=x_{t}^{*}\left(u_{0}\right)$, since $w-\lim _{n \rightarrow \infty} u_{n}=u_{0}$. Hence,

$$
\lim _{n \rightarrow \infty}\left(f u_{n}\right)(s)=\left(f u_{0}\right)(s), \quad s \in\left[0, t_{0}\right]
$$

Therefore, by Lebesgue's dominated convergence theorem, we obtain (18). From (14), (15) and (18), it follows that $x_{n}\left(t_{n}\right) \rightarrow x_{0}\left(t_{0}\right)=\hat{x} \in W$.

Now we deal with the time-optimal control if the target set $W$ is a singleton. Let $W=w_{0}$ such that $\phi^{0} \neq w_{0}$ and $\phi^{1}(s) \neq w_{0}$ for some $s \in[-h, 0[$. Then we can choose a decreasing target set $\left\{W_{n}\right\}$ of convex and weakly compact sets with a nonempty interior satisfying

$$
w_{0} \in \bigcap_{n=1}^{\infty} W_{n}, \text { and } \operatorname{dist}\left(w_{0}, W\right)=\sup _{x \in W_{n}} E\left|x-w_{0}\right| \rightarrow 0(n \rightarrow \infty) .
$$

Define

$$
U_{0}^{n}=\left\{u \in U_{a d}: x_{u}(t) \in W_{n} \text { for some } t \in[0, T]\right\} .
$$

Then, we may consider that $u_{n}$ is the time-optimal control with the optimal time $t_{n}$ to the target set $W_{n}, n=1,2, \ldots$

Theorem 2. Let Assumption (G) be satisfied. Suppose that $\left\{W_{n}\right\}$ is a sequence of closed convex and weakly compact sets in $H$ satisfying (19) and $U_{0}^{n} \neq \varnothing$. Then there is a time-optimal control $u_{0}$ with the optimal time $t_{0}=\sup _{n \geq 1}\left\{t_{n}\right\}$ to the singleton $\left\{w_{0}\right\}$, which is defined by the weak limit of some subsequence of $\left\{u_{n}\right\}$ in $L^{2}\left(0, t_{0} ; U\right)$.

Proof. Let $w_{n}=x_{n}\left(t_{n}\right) \in W_{n}$. Because of the weak compactness of $U_{a d}$, there are $u_{0} \in U_{a d}$ and subsequences (which are denoted again by $\left\{u_{n}\right\}$ and $\left\{w_{n}\right\}$ ) such that $t_{n} \uparrow t_{0}$ as $n \rightarrow \infty$, $u_{n} \rightarrow u_{0}$ weakly in $L^{2}\left(0, t_{0} ; U\right)$, and $w_{n}=x_{n}\left(t_{n}\right) \in W_{n} \rightarrow w_{0}$ strongly in $H$. Then by virtue of (19), we have

$$
E\left|w_{n}-w_{0}\right| \rightarrow 0
$$

strongly in $H$. Thus, in the similar argument as the proof of Theorem 2, we can easily prove that $u_{0}$ and $t_{0}$ are the time-optimal control and the optimal time to the target $\left\{w_{0}\right\}$, respectively.

Example 1. Let

$$
H=L^{2}(0, \pi), V=H_{0}^{1}(0, \pi), V^{*}=H^{-1}(0, \pi),
$$

and let $U$ be a Banach space of control variables. Consider the following control system, which is described as a retarded neutral stochastic differential system on $H$ :

$$
\left\{\begin{aligned}
\partial x(t, y)= & {\left[\Delta x(t, y)+\int_{-h}^{0} a(s) \Delta x(t+s, y) d s+B u(t, y)\right] \partial t } \\
& +f\left(t, x_{t}\right) d \omega(t), \quad(t, y) \in[0, T] \times[0, \pi] \\
x(0, y)= & \phi^{0}(y) \in L^{2}(\Omega, H), x(s, y)=\phi^{1}(s, y), \quad(s, y) \in[-h, 0) \times[0, \pi]
\end{aligned}\right.
$$


where $h>0, a(\cdot)$ is Hölder-continuous, and $\omega(t)$ stands for a standard cylindrical Winner process in $H$ defined on a stochastic basis $(\Omega, \mathcal{F}, P)$. Define the sesquilinear form $b(\cdot, \cdot)$ on $V \times V$ by

$$
b(u, v)=\int_{0}^{\pi} \frac{d u(y)}{d y} \frac{\overline{d v(y)}}{d y} d y,
$$

satisfying Gärding's inequality (2). Let

$$
A=\partial^{2} / \partial y^{2} \quad \text { with } \quad D(A)=\left\{x \in H^{2}(0, \pi): x(0)=x(\pi)=0\right\} .
$$

Then the eigenvalue of $A=\Delta$ is $\lambda_{n}=-n^{2}$, and its eigenfunction is given by $z_{n}(y)=(2 / \pi)^{1 / 2} \sin n y$. Since $\left\{z_{n}: n \in N\right\}$ is an orthogonal basis of $H$, the semigroup $S(t)$ generated by $A$ is represented as

$$
S(t) x=\sum_{n=1}^{\infty} e^{n^{2} t}\left(x, z_{n}\right) z_{n}, \quad \forall x \in H, t>0
$$

and there is a positive constant $M_{0}$ such that $\|S(t)\|_{\mathcal{L}(H)} \leq M_{0}$. For any $\left.x \in \Pi\right)$, set

$$
\left.f\left(t, x_{t}\right)\right)=\int_{0}^{t} \sum_{i=1}^{n} \frac{\partial}{\partial x_{i}} \sigma_{i}\left(t, \nabla x_{t}\right) d s .
$$

We assume that there is a constant $L>0$ such that

$$
\left|\sigma_{i}(t, \xi)-\sigma_{i}(t, \widehat{\xi})\right| \leq L|\xi-\widehat{\xi}|,
$$

where $|\cdot|$ is the norm of $H=L^{2}(\Omega)$. For simplicity, we assume that $\sigma_{i}(t, 0)=0$. Hence, we have

$$
\left|\sigma_{i}(t, \xi)\right| \leq L|\xi| .
$$

Put

$$
g_{1}\left(t, x_{t}\right)=\sum_{i=1}^{n} \frac{\partial}{\partial x_{i}} \sigma_{i}\left(t, \nabla x_{t}\right)
$$

Then $g_{1}(t, x) \in V^{*}$. For each $w \in H_{0}^{1}(\Omega)$, we satisfy the following that

$$
\left(g_{1}\left(t, x_{t}\right), w\right)=-\sum_{i=1}^{n}\left(\sigma_{i}\left(t, \nabla x_{t}\right), \frac{\partial}{\partial x_{i}} w\right) .
$$

The nonlinear term is given by

$$
f\left(t, x_{t}\right)=\int_{0}^{t} g_{1}\left(t, x_{t}\right) d s .
$$

For any $w \in H_{0}^{1}(\Omega)$, if $u$ and $\hat{u}$ belong to $H_{0}^{1}(\Omega)$, by (21) we obtain

$$
E\left|\left(g\left(t, x_{t}(s)\right)-g\left(t, \hat{x}_{t}(s)\right)\right), w\right| \leq L T E|| x_{t}(s)-\hat{x}_{t}(s)||\|w\| .
$$

Thus, it is easily seen that Assumption (G) has been satisfied. Define $B \in \mathcal{L}(U, H)$ by

$$
B u(t)=\gamma(y) u, \quad 0 \leq y \leq \pi, u \in L^{2}(0, T ; U), \gamma(y) \in M^{2}([0, \pi]) .
$$

Let $U$ be a real Banach space and let the admissible set $U_{a d}$ be a weakly compact subset in $L^{2}(0, T ; U)$. If $U_{0}$ is nonempty, then by Theorem 2 , there is a control $\bar{u} \in U_{0}$ such that

$$
\tilde{t}(\bar{u}) \leq \tilde{t}(u) \quad \text { for all } u \in U_{0}
$$

governed to the constraint (20). 


\section{Conclusions}

Most previous optimal control theories typically state that the main operators are bounded linear. However, little study has been done of the existence of time-optimal control for control to a target set for nonlinear stochastic functional differential equations with unbounded operators, which is not able to obtain the regularity of the solution since the integral of $A S(t-s)$ has a singularity at $t=s$ (see Lemma 1 ). As we get the boundedness and inequalities in Lemma 2 by configuring and interpreting the fundamental solution for linear functional equations with time delays as seen in [22], we can interpret the regularity of the solution. Moreover, by the basic consequence of real interpolation spaces and establishing variations of constant formulae of solutions, we investigate the time-optimal control problem to a bounded target set for semilinear stochastic control equations involving time delays or memories, although the equations contain unbounded principal operators and nonlinear terms. The presence of time-optimal controls only for a set of one-point targets governed by the given semilinear stochastic equation is also established. Based on this approach, we intend to study the approaches and applicable methods of various nonlinear stochastic equations in science.

Author Contributions: Conceptualization, Y.H.K. and J.-M.J.; formal analysis, Y.H.K.; methodology, J.-M.J.; writing-review-editing, J.-M.J. All authors have read and agreed to the published version of the manuscript.

Funding: This research received no external funding.

Institutional Review Board Statement: Not applicable.

Informed Consent Statement: Not applicable.

Data Availability Statement: Not applicable.

Conflicts of Interest: The authors declare no conflict of interest.

\section{References}

1. Bharucha-Ried, A.T. Random Integral Equation; Academic Press: New York, NY, USA, 1982.

2. Grecksch, W.; Tudor, C. Stochastic Evolution Equations: A Hilbert Space Apprauch; Academic: Berlin, Germany, 1995.

3. Katayama, T. A history of symposium on stochastic systems theory and its applications. IFAC J. Syst. Control 2020, 11, 1-6.

4. Metivier, M.; Pellaumail, J. Stochastic Integration; Academic Press: New York, NY, USA, 1980.

5. Balasubramaniam, P. Existence of solutions of functional stochastic differential inclusions. Tamkang J. Math. 2002, 33, 35-43. [CrossRef]

6. Curtain, R.F. Stochastic evolution equations with general white noise disturbance. J. Math. Anal. Appl. 1977, 60, 570-595. [CrossRef]

7. Balasubramaniam, P.; Park, J.Y.; Muthukumar, P. Approximate controllability of neutral stochastic functional differential systems with infinite delay. Stoch. Anal. Appl. 2010, 28, 389-400. [CrossRef]

8. Ren, Y.; Hu, L.; Sakthivel, R. Controllability of neutral stochastic functional differential inclusions with infinite delay. J. Comput. Appl. Math. 2011, 235, 2603-2614. [CrossRef]

9. Yang, M.; Wang, Q. Approximate controllability of Caputo fractional neutral stochastic differential inclusions with state-dependent delay. IMA J. Math. Control Inform. 2018, 35, 1061-1085. [CrossRef]

10. Zhang, R.; Guo, L. Controllability of stochastic game-based control systems. SIAM J. Control Optim. 2019, 57, 3799-3826. [CrossRef]

11. Mahmudov, N.I. Approximate controllability of semilinear deterministic and stochastic evolution equations in abstract spaces. SIAM J. Control Optim. 2006, 42, 175-181. [CrossRef]

12. Sakthivel, R.; Mahmudov, N.I.; Lee, S.G. Controllability of non-linear impulsive stochastic systems. Internat. J. Control 2009, 82, 801-807. [CrossRef]

13. Nakagiri, S.I. Optimal control of linear retarded systems in Banach spaces. J. Math. Anal. Appl. 1986, 120, 169-210. [CrossRef]

14. Mordukhovich, B.S.; Wang, D.; Wang, L. Optimal control of delay-differential inclusions with functional endpoint constraints in infinite dimensions. Nonlinear Anal. 2009, 71, 2740-2749. [CrossRef]

15. Mordukhovich, B.S.; Wang, D.; Wang, L. Optimization of delay-differential inclusions in infinite dimensions. Pac. J. Optim. 2010, 6, 353-374.

16. Fattorini, H.O. Time and norm optimal controls:a survey of recent results and open problems. Acta Math. Sci. Ser. B (Engl. Ed.) 2011, 31, 2203-2218. 
17. Hafayed, D.; Chala, A. An optimal control of a risk-sensitive problem for backward doubly stochastic differential equations with applications. Random Oper. Stoch. Equ. 2020, 28, 1-18. [CrossRef]

18. Micu, S.; Roventa, I.; Tucsnak, M. Time optimal boundary controls for the heat equation. J. Funct. Anal. 2012, 263, 25-49. [CrossRef]

19. Krakowiak, A. Time optimal control of retarded parabolic systems. IMA J. Math. Control Inform. 2007, 24, 357-369. [CrossRef]

20. Tanabe, H. Equations of Evolution; Pitman: London, UK, 1979.

21. Jeong, J.M.; Son, S.J. Time Optimal Control of Semilinear Control Systems involving Time Delays. J. Optim. Theory Appl. 2015, 165, 793-811. [CrossRef]

22. Tanabe, H. Fundamental solutions for linear retarded functional differential equations in Banach space. Funkcial. Ekvac. 1992, 35, 149-177.

23. Triebel, H. Interpolation Theory, Function Spaces, Differential Operators; Verlag der Widdendchaften: Berlin, Germany, 1978.

24. Nakagiri, S. Controllability and Identifiability for Linear Time-Delay Systems inHilbert Space; Lecture Notes in Control and Information Sciences; Springer: Berlin, Germany, 1991; Volume 159.

25. Jeong, J.M. Stabilizability of retarded functional differential equation in Hilbert space. Osaka Math. J. 1991, $28,347-365$.

26. Kang, H.K.; Jeong, J.M. $L^{2}$-primitive process for retarded stochastic neutral functional differential equations in Hilbert spaces. J. Comput. Anal. Appl. 2021, 29, 838-861.

27. Yong, J.; Pan, L. Quasi-linear parabolic partial differential equations with delays in the highest order partial derivatives. J. Aust. Math. Soc. 1993, 54, 174-203. [CrossRef]

28. Nakagiri, S. Structural properties of functional differential equations in Banach spaces. Osaka Math. J. 1988, 25, 353-398. 